\title{
Curvas de altura dominante e índice de sitio en plantaciones de Prosopis alba
}

\author{
Senilliani, M. G., Bruno, C. y Brassiolo, M.
}

DOI: $10.31047 / 1668.298 x . v 38 . n 2.30940$

\begin{abstract}
RESUMEN
Se definieron y validaron curvas de altura dominante e índice de sitio que permitieron inferir las calidades del sitio forestal del algarrobo blanco y caracterizar los factores del sitio que tengan mayor influencia en el crecimiento de la especie en el área de riego de la provincia de Santiago del Estero, Argentina. A partir del modelo Gompertz se ajustó una función promedio para la altura dominante-edad mediante el método de la curva guía, de la cual se derivó la familia de curvas por el principio de proporcionalidad constante. La tendencia muestra que el crecimiento en altura culmina próximo a los 20 años. Una primera aproximación en la estimación de la capacidad productiva de los sitios para Prosopis alba Griseb. se expresa a partir de la familia de curvas anamórficas a la edad base de 15 años que describen tres calidades: I (buena), II (regular) y III (mala). Con relación a los factores del sitio, las propiedades químicas evaluadas en suelos fueron determinantes en el crecimiento, condicionado negativamente por la conductividad eléctrica y demás parámetros característicos de las condiciones de salinidad. El proceso de validación determinó un error entre 5 y $10 \%$.
\end{abstract}

Palabras clave: algarrobo blanco, calidad de sitio, forestación, modelos no lineales

Senilliani, M. G., Bruno, C. and Brassiolo, M., 2021. Dominant height curves and site index in Prosopis alba plantations. Agriscientia 38 (2): $13-25$

\section{SUMMARY}

Dominant height curves and site index were defined and validated, which allowed inferring the qualities of the forest site of the white carob tree and characterizing the most influential site factors on the growth of the species in the irrigated area in the province of Santiago del Estero, Argentina. Based on the Gompertz model, an average function was adjusted for the dominant heightage by the guide curve method, from which the family of curves was derived by the principle of constant proportionality. The trend shows that the growth in height culminates close to 20 years. A first approximation in the estimation 
of the productive capacity of the sites for Prosopis alba Griseb. is expressed from the family of anamorphic curves at the base age of 15 years that describe three qualities: I (high), II (medium) and III (low). In relation to site factors, the chemical properties evaluated in soils were decisive in growth, negatively conditioned by electrical conductivity and other characteristic parameters of salinity conditions. The validation process determined an error between 5 and $10 \%$.

Key words: algarrobo blanco, site quality, afforestation, nonlinear models

Senilliani, M. G. (ORCID: 0000-0003-3570-3091) y Brassiolo, M. (ORCID: 0000-0003-2845-8390): Universidad Nacional de Santiago del Estero, Facultad de Ciencias Forestales, INSIMA. Av. Belgrano (S) 1912, Santiago del Estero, Argentina. Bruno, C. (ORCID: 00000002-3674-7128): Universidad Nacional de Córdoba, Facultad de Ciencias Agropecuarias. Av. Félix Marrone 746, Ciudad Universitaria, CC509, CP 5000. Córdoba, Argentina. Consejo Nacional de Investigaciones Científicas y Técnicas (CONICET), Unidad de Fitopatología y Modelización Agrícola (UFyMA), Córdoba, Argentina. Correspondencia a: senilliani@yahoo.com.ar

\section{INTRODUCCIÓN}

El género Prosopis tiene gran relevancia entre la flora presente en la mayor parte de la Región Chaqueña de Argentina. Entre las especies del género se destaca $P$. alba como una especie nativa de gran importancia (Giménez et al., 2001; Cisneros y Moglia, 2017) debido a que constituye una de las principales especies nativas de Argentina como fuente de madera para aserrío. Las estadísticas confirman que de las tres especies más usadas para este destino, el algarrobo blanco ocupó el primer lugar en importancia con un $19 \%$ de la producción total de madera proveniente de especies nativas en el país (Dirección Nacional de Desarrollo Foresto Industrial, Ministerio de Agroindustria de la Nación Argentina, 2015).

En la región del noroeste argentino, se conducen investigaciones tendientes a dar respuesta a los problemas que se generan en el cultivo forestal de esta especie en aspectos relacionados al mejoramiento genético, tratamientos silviculturales y sanitarios en vivero y plantación (Ledesma et al., 2008; Navall et al., 2015; Salto et al., 2016). La reforestación con algarrobo en la Región Chaqueña y en la provincia de Santiago del Estero es una actividad que sigue creciendo, constituyendo en la región el eje de la industria de la fabricación de muebles dada su importancia económica y social.
La especie presenta una fácil adaptación a los sitios de reforestación pero una alta variabilidad intrínseca sumada a las diferencias de crecimiento en respuesta a las diferencias en la calidad de los sitios, siendo esta situación común en la región (Kees et al., 2017). Para el fomento y la consolidación de la actividad forestal es necesario implementar una zonificación basada en la evaluación de los sitios potenciales para $P$. alba y así determinar y/o crear las condiciones para el establecimiento de reforestaciones, concentrando la producción en áreas de alto potencial. Este enfoque silvícola requiere de una base amplia de conocimientos que permitan entender y caracterizar la dinámica y productividad de la especie. En plantaciones forestales cuyo propósito es la explotación maderera, la calidad de sitio se define como su potencial para la producción de madera de una especie o un tipo de bosque, considerando que mejores calidades de sitio tendrán mayor producción (Clutter et al., 1983; Senilliani et al., 2019). El concepto de calidad del sitio hace referencia a la influencia del ambiente sobre la producción de un determinado servicio ecosistémico del bosque, ya sea un servicio de provisión como puede ser madera, forraje y/o frutos; un servicio de regulación que puede estar abocado a la regulación del agua o protección del suelo, o de soporte (conservación de la vida silvestre que los habita y la biodiversidad) y/o 
cultural relacionado a la valoración estética y de recreación (Schlatter y Gerding, 2014).

La productividad es un concepto biológico, en consecuencia su valor o magnitud carece de representación numérica; es por este motivo que la magnitud de la productividad de un bosque puede ser representada a través de la calidad de sitio que construye su medida de valor a través de indicadores que en su conjunto conforman un índice denominado índice de sitio (Prodan et al., 1997). Se define el término índice de sitio dasométrico (IS) como el valor numérico dado por la altura dominante $(\mathrm{Hd})$ de un rodal a una edad determinada, llamada edad base (Alder, 1980) y se emplea como expresión de la calidad de sitio (Senilliani et al., 2019).

Diversos estudios han utilizado el IS para evaluar la productividad de las especies forestales. En la región mesopotámica de Argentina, Caniza y Torrez (2019), Caniza et al. (2016) y Crechi et al. (2011) son algunos de los autores que han realizado estudios de crecimiento de la altura dominante en plantaciones de coníferas y latifoliadas de rápido crecimiento. En 2011 Crechi et al. compararon tres métodos tradicionales para determinar un índice de sitio para Eucalyptus grandis en dos regiones agroecológicas: uno fue a través del uso de la curva guía, otro basado en la reparametrización del modelo y un tercer método considerando ecuaciones diferenciales. El procedimiento elegido dependió del método de la calidad de información disponible en las zonas agroecológicas de estudio. En la provincia de Chaco, Kees et al. (2017) trabajaron con curvas de crecimiento en altura dominante con el propósito de determinar el modelo de índice de sitio para P. alba. Para ello se ajustó el modelo Gompertz, y se aplicó el método de la curva guía con la hipótesis de crecimiento anamórfico.

En la provincia de Santiago del Estero, inserta en la Región Chaqueña, fueron realizados algunos estudios de crecimiento en $P$. alba en formaciones boscosas nativas. Juárez de Galindez et al. (2005) realizaron la modelación del crecimiento en algarrobo blanco empleando dos modelos biológicos mediante el análisis del fuste en individuos de monte nativo. En lo que respecta a plantaciones, se han realizado importantes avances; sin embargo, aún no se han desarrollado curvas de calidad de sitio para plantaciones de $P$. alba en el área de producción bajo riego, donde en la actualidad se concentran la mayoría de las reforestaciones con esta especie. La clasificación de los sitios es un paso fundamental en el manejo de rodales y bosques, por lo cual se considera que si se aplicaran curvas de altura dominante e índice de sitio en el manejo silvícola de la especie en estudio representaría una herramienta básica para estimar con precisión el crecimiento y la producción futura; a la vez que conocer los factores del sitio que favorecen y limitan su desarrollo, permitirá predecir la calidad de determinados sitios donde aún no existen plantaciones. La hipótesis del estudio es que la evolución de la altura dominante en rodales de $P$. alba en el área de riego en la provincia de Santiago del Estero tiene un comportamiento de carácter anamórfico, que puede ser descripto a partir de las curvas anamórficas que darán origen a las diferentes clases de calidad de sitio, donde el grado de salinidad es uno de los factores de mayor impacto en el crecimiento.

El objetivo de este trabajo es establecer un modelo que permita definir las curvas de altura dominante e índice de sitio (IS) a partir de las cuales se pueda inferir las calidades del sitio forestal y poder caracterizar los factores del sitio que tengan más influencia en el crecimiento de la especie implantada en sistemas silvícolas en áreas de riego.

\section{MATERIALES Y MÉTODOS}

El área de estudio se sitúa en el Complejo Valle del Río Dulce dentro de la Subregión del Chaco Semiárido de la República Argentina. Está comprendida dentro del área de riego del Río Dulce en la provincia de Santiago del Estero, a $27^{\circ} 39^{\prime}$ S, $64^{\circ} 14^{\prime} \mathrm{O}$ (Morello, 2012) (Figura 1). El clima se caracteriza por ser subtropical con estación seca, con déficit hídrico y variabilidad climática dentro del ciclo anual y entre períodos anuales. El área en estudio presenta una temperatura promedio anual de $20,4{ }^{\circ} \mathrm{C}$ y precipitación promedio anual de $624 \mathrm{~mm}$. Según datos de las estaciones meteorológicas situadas en el Aeropuerto Santiago del Estero y en el INTA La Abrita, ambas ubicadas en la zona de estudio, se corrobora la información presentada, en lo que respecta a precipitación promedio anual con valores entre 500 a $700 \mathrm{~mm}$ aproximadamente a partir de datos históricos (1961-2017), distribuida a través del año bajo un régimen estival. La estación lluviosa se extiende de octubre a marzo y el resto de los meses comprende la estación seca; se clasifica el área como subhúmeda-seca, la temperatura del mes más cálido es de $26,3{ }^{\circ} \mathrm{C}$ y la del mes más frío $11,9^{\circ} \mathrm{C}$ (Morello, 2012).

El área donde se ubican actualmente los rodales de algarrobo blanco en la provincia corresponde a la Llanura aluvial del río Dulce, Paleo Llanura de Inundación, con material originario fluvio-loéssico, 
que posee gran heterogeneidad espacial en cuanto a las propiedades químicas de los suelos principalmente. Según la información registrada a través de GeolNTA (2017) a partir del mapa de suelos de la República Argentina basada en la clasificación taxonómica SoilTaxonomy (Soil Survey Staff, 2014), la mayor parte de las áreas con plantaciones se ubican en suelos MNai-7 y 10 (Haplustoles arídicos), los que se caracterizan por presentar perfiles de textura franca a francoarenosa, con baja humedad, no sódicos. Algunos rodales se ubican en suelos Ektc-12 (Haplustoles salortídicos) de texturas arenosas con algún tipo de limitante de salinidad en los $50 \mathrm{~cm}$ de profundidad y generalmente ubicados en vías de escurrimiento plano cóncavas, y en AEtc-34 (Natracualfes típicos) de texturas franco-arenosas, de drenaje deficiente, de plano aluvial. El área de estudio en general se caracteriza por registrar valores de conductividad eléctrica que varían de 5 a $20 \mathrm{dSm}^{-1} \mathrm{y}$ concentración de sodio del extracto de saturación en un rango de 21 a $137 \mathrm{meg} / \mathrm{l}$. La presencia de la capa freática se encuentra cercana entre los
2 y $5 \mathrm{~m}$ de profundidad, aproximadamente.

Las áreas de influencia de este estudio comprenden bosques y vegetación dentro de la cuenca del Río Dulce, que adopta características distintivas con respecto a la subregión del semiárido. Se presentan comunidades riparias, con la presencia de los géneros y especies Aspidosperma quebracho-blanco Schltdl., Lycium spp., Schinopsis spp., Prosopis sericantha Gill. Ex Hook \&Arn. y $P$. alba Griseb. Se trata de un fachinal abierto dominado por Vachellia aroma ex Hook. \& Arn. (tusca), Celtis iguanaea (Jacq.) Sarg. (tala), con árboles aislados de Schinopsis lorentzii Griseb (quebracho colorado) y Sarcomphalus mistol Griseb (mistol) (Sarmiento, 1963; Red Agroforestal Chaco Argentina [RedAF], 1999).

En este estudio el criterio de selección de las plantaciones fue una combinación de la edad y la variabilidad en los tipos de suelos, teniendo en cuenta principalmente la salinidad y las características propias de los rodales, buscando maximizar la variabilidad entre muestras y

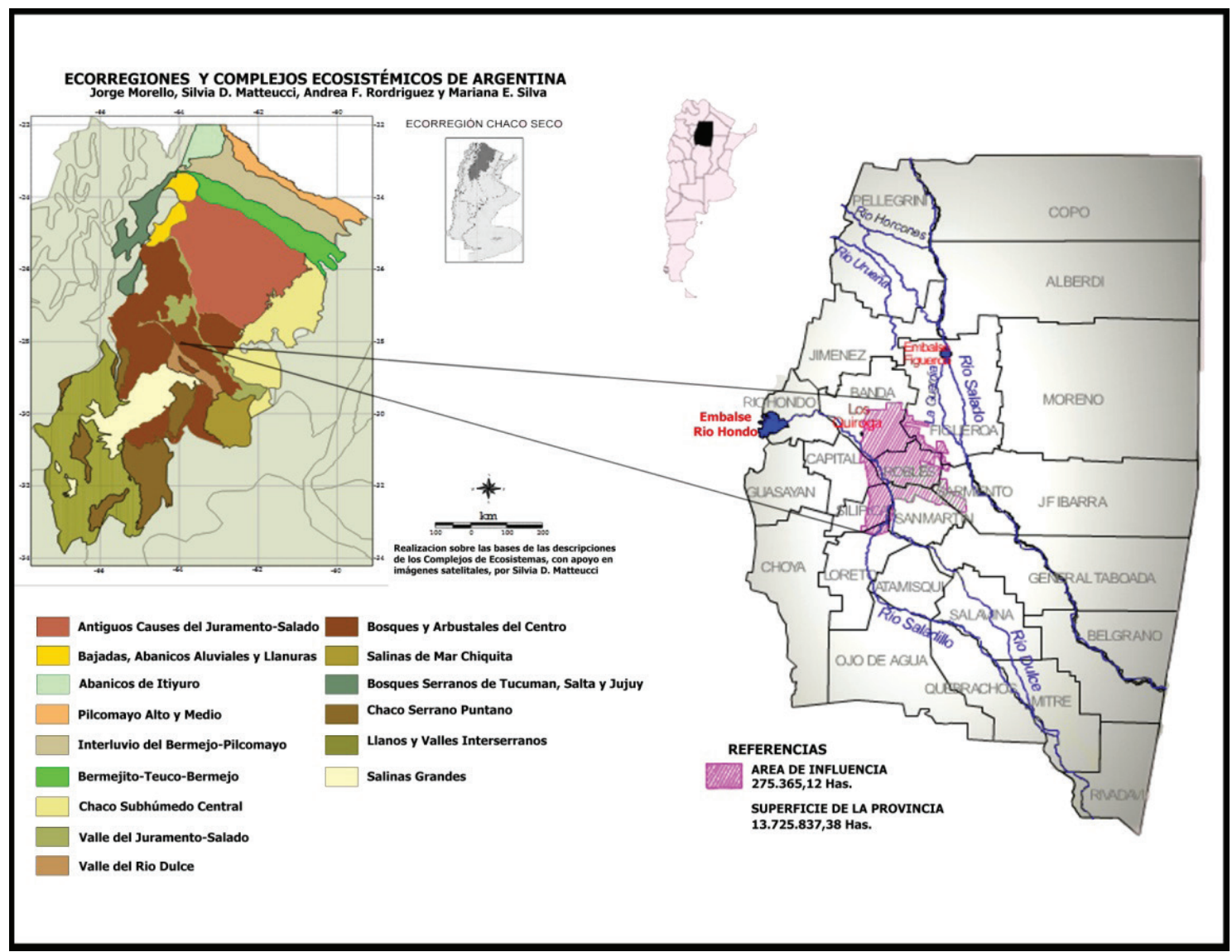

Figura 1. Mapa de la provincia de Santiago del Estero, localizado en la ecorregión del Chaco Seco, República Argentina. Fuente: Morello et al. (2012) y Unidad Ejecutora del servicio de riego del río Dulce. Elaboración propia 
minimizar la variabilidad dentro de la unidad de muestreo. Cabe aclarar que se relevaron todas las plantaciones que superaron la edad mínima establecida de 9 años. Para evaluar la variabilidad se calculó el coeficiente de variación $(\mathrm{CV})$ de la altura dominante $(\mathrm{Hd})$, que resultó en un valor máximo de $25 \%$ de variación dentro de la unidad muestral (Herrera, 1996). Se trabajó con un total de 26 parcelas ubicadas en las diferentes plantaciones del área de riego. Cada parcela posee un número fijo de 48 árboles y por consiguiente representan un área variable entre 576 a $1728 \mathrm{~m}^{2}$, según el espaciamiento inicial de la plantación. A la fecha de plantación de estos rodales no se contaba con material genético mejorado, por lo cual el origen del germoplasma empleado provino en su totalidad de poblaciones locales. La base de datos a utilizar provino de 11 parcelas temporales (PT), o sea aquellas parcelas donde se ha medido la variable de crecimiento solo una vez; 15 parcelas permanentes (PP), o sea parcelas donde se ha medido la variable de crecimiento en medidas sucesivas en el tiempo, en algunos casos 2, 3 o 4 mediciones; y se han obtenido datos de crecimiento provenientes del análisis epidométrico de 11 árboles dominantes extraídos en dichas plantaciones. Se dispuso de 91 pares de datos altura-edad, incluyendo datos del análisis fustal de los árboles seleccionados para el análisis epidométrico. Las muestras para el análisis fustal se extrajeron de cada individuo seleccionando tortas de $5 \mathrm{~cm}$ de espesor a las siguientes alturas desde la base: 0,30 m, 1,3 m, $2,0 \mathrm{~m}$ y $3,0 \mathrm{~m}$. Estas fueron preparadas en la forma descripta por Giménez et al., (1998) y luego digitalizadas con escáner HP Scanjet G2410, a una resolución de 1200 dpi para la medición de los anillos de crecimiento mediante el software IPWIN 4. Se trabajó sobre cuatro radios perpendiculares a partir de los que se consignó el número de años y el espesor del anillo con una precisión de centésima de milímetro.

Para la caracterización del sitio se hizo un relevamiento preliminar del suelo en cada una de las parcelas. El estudio del perfil se realizó con calicatas situadas en el centro de cada parcela, extrayendo muestras de 0 a $90 \mathrm{~cm}$ de profundidad, discriminando por horizontes. Los análisis de las muestras de suelo se realizaron en el laboratorio del INSIMA, de la Facultad de Ciencias Forestales en la Universidad Nacional de Santiago del Estero (FCF-UNSE) y en el Laboratorio de Suelo, Agua y Fertilizantes (LabSA) de la Estación Experimental Agropecuaria Salta, Grupo Recursos Naturales. Los parámetros edáficos físicos determinados fueron densidad aparente (DA) $\left(\mathrm{g} / \mathrm{cm}^{3}\right)$ por el método del cilindro y porosidad de la capa arable (PTot) por el método de gravimetría de muestras volumétricas $\left(100 \mathrm{~cm}^{3}\right)(\% \mathrm{v} / \mathrm{v})$ (Klute, 1986). Los parámetros edáficos químicos se determinaron de acuerdo a las siguientes técnicas: pH por potenciometría, conductividad eléctrica (CE) estandarizada a $25^{\circ} \mathrm{C}\left(\mathrm{dSm}^{-1}\right)$, Sodio $\left(\mathrm{Na}^{+}\right)$por fotometría de llama y Calcio más Magnesio $(\mathrm{Ca}+\mathrm{Mg})$ por volumetría con EDTA (meq/l), todos sobre el extracto de saturación (Sparks, 1996) y presencia de carbonatos (\%) mediante método descriptivo de reacción con $\mathrm{HCl}$ al 10 \% según la guía de reconocimiento de suelos de la Organización de las Naciones Unidas para la Alimentación y la Agricultura (FAO, 2009). La presencia de carbonatos se categorizó en una escala ordinal donde 0 indicó la ausencia de carbonatos en todo el perfil; 1 representó la presencia de carbonatos en concentraciones entre $0-2 \%$ en la profundidad del perfil a partir de los $70 \mathrm{~cm}$ de profundidad; 2 representó la presencia de carbonatos concentraciones entre 2 a $10 \%$ también a partir de los $70 \mathrm{~cm}$ de profundidad, y 3 la presencia de carbonatos en todo el perfil con concentraciones entre 10 a $25 \%$. Se calculó la relación adsorción sodio (RAS) tomando la base general de datos con la siguiente formula $\left.\mathrm{RAS}=\mathrm{Na}^{+} / \mathrm{raíz}((\mathrm{Ca}+\mathrm{Mg}) / 2)\right)$.

En la evaluación del desarrollo de los rodales las variables medidas fueron: el diámetro a la altura de pecho (DAP) expresado en centímetros (cm) y medido con cinta diamétrica; la altura total del árbol $(H T)$, expresada en metros $(m)$ y medida con vara telescópica, y la edad (E) que fue expresada en años. En el análisis epidométrico, el espesor de anillos de crecimiento fue expresado en milímetros $(\mathrm{mm})$. Para la determinación del índice de sitio se consideró la altura dominante $(\mathrm{Hd})$ definida como la altura promedio de los 100 árboles más gruesos por hectárea (Assmann, 1970) expresada en metros $(\mathrm{m})$.

Para la determinación de las curvas de altura dominante, segenerólaserieedad-altura dominante construida a partir de registros dasométricos de las parcelas temporales y permanentes de medición y de los datos del análisis epidométrico de los fustes de árboles seleccionados. Se procedió según el método de curva guía que consistió en determinar una única función promedio a partir de la serie de datos altura dominante-edad y sobre la base de esta, derivar las demás curvas por un principio de proporcionalidad constante (Barrero-Medel et al. 2011; Hernández-Ramos et al., 2014).

Se utilizó el modelo matemático de Gompertz sugerido por Senilliani et al. (2019) como el más adecuado para la especie, un modelo de regresión no lineal mixto de Gompertz, con correlación por 
simetría compuesta y varianza de los errores con función de varianza exponencial. A partir de la curva guía y considerando la edad base determinada se trazaron las curvas de igual forma o curvas anamórficas, que pasaron por diferentes valores de IS; a este proceso se lo denomina armonización (Alder, 1980; Prodan et al, 1997). Se generó la familia de curvas anamórficas haciendo variar la edad y el IS deseado para la edad base (Eb) predeterminada (Andenmatten y Letourneau, 1998; Mares Arreola et al., 2004; Barrero-Medel et al., 2011; Hernández-Ramos et al., 2014). El procedimiento de armonización se realizó teniendo en cuenta la fórmula de Gompertz [Ecuación 1] para modelar la Hd en función de la edad base y en función de la definición de índice de sitio [Ecuación 2]. Las ecuaciones del proceso de armonización son las siguientes:

$$
\begin{aligned}
& \mathrm{Hd}=\alpha \cdot \mathrm{e}^{\left(-\beta \cdot \mathrm{e}^{(-\gamma \cdot \mathrm{E})}\right)} \\
& \mathrm{IS}=\alpha \cdot \mathrm{e}^{\left(-\beta . \mathrm{e}^{(-\gamma . \mathrm{E} \text {.Ed base }))}\right.}
\end{aligned}
$$

el parámetro a se despeja de la ecuación anterior [2] de la siguiente manera:

$$
\alpha=\frac{I S}{e^{\left(-\beta . e^{(-\gamma . E d a d ~ b a s e)}\right.}}
$$

Luego, reemplazando en la Ecuación [1] se tiene que:

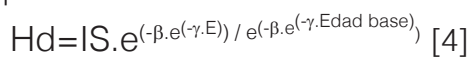

En resumen, la familia de curvas anamórficas, se obtuvo despejando $\alpha$ en la ecuación de índice de sitio y sustituyendo la expresión resultante en la ecuación original [1], con lo que el valor asintótico se consideró implícito y se asumió que los parámetros de forma son comunes para todos los sitios (Hernandez-Ramos et al., 2014). A partir de la dispersión de los datos de altura dominante obtenidos a la edad base se determina la equidistancia entre las curvas de IS. La validación del modelo de predicción del índice de sitio se realizó a través de remuestreos con reposición técnica denominada Bootstrap (Efron y Tibshirani, 1993). A partir de los remuestreos se estimó el promedio de la raíz cuadrada del error (RECM) calculado como los desvíos al cuadrado. En cada clase de sitio establecida se calculó el promedio de las alturas observadas para cada año de edad y con estos datos se estimaron los índices de sitio (ISest). Posteriormente se calculó el error respecto al índice de sitio para cada clase, (ISobs) (Barret, 1978; Fassola y Wabo, 1993 y Andenmatten y Letourneau, 1998).

Para realizar la caracterización de los factores edáficos que determinan la calidad de sitio se hizo una evaluación preliminar de la relación de la Hd y las variables edáficas relevadas en las parcelas. Se asignó a cada una de las parcelas de estudio su correspondiente clase de calidad a través del cálculo del IS, utilizando la altura dominante y la edad actual con la función del IS descripta en el ítem anterior. Se realizó un análisis de la varianza multivariado (MANOVA) para evaluar diferencias entre clases de calidad de sitio considerando las propiedades físicas y químicas. La variable Carbonatos fue analizada mediante tablas de contingencia según las categorías descriptas anteriormente; se trabajó con el estadístico Chi cuadrado para analizar las diferencias estadísticas entre clases de calidad.

La determinación de diferencias entre las clases de calidad de sitio para la variable IS y Hd se realizó mediante ANOVA. Si bien se analizaron las muestras de suelo de cada horizonte del perfil, se tomó el primer horizonte (A) de referencia para los análisis estadísticos por ser el que mejor permitió diferenciar calidades de sitio. Los datos fueron acondicionados, depurados y analizados estadísticamente con InfoStat (Di Rienzo et al., 2020) y su interface con el programa $R$ ( $R$ CoreTeam, 2020).

\section{RESULTADOS Y DISCUSIÓN}

Se registraron los valores promedios de altura dominante según clase de calidad e IS con diferencias estadísticamente significativas entre clases en la $\mathrm{Hd}(F=19,5 ; p<0,0001)$ e IS $(F=53,95 ; p<0,0001)$. Los resultados del análisis de la varianza confirman que se encuentran correctamente representadas las tres calidades como resultado del crecimiento en altura de los rodales en estudio. Las muestras responden en $26,6 \%$ a la mejor calidad de sitio (calidad I), 55,5\% a la calidad regular (calidad II) y $17,7 \%$ a la menor calidad (calidad III).

Los valores obtenidos indicaron que la $\mathrm{Hd}$ se incrementa con la edad a una tasa mayor en la etapa inicial de la vida del rodal que en la madurez y se registraron mayores coeficientes de variación en los rodales de menor edad con respecto a los adultos (71\% vs. $13 \%)$. El proceso de crecimiento se describe en tres etapas: una etapa inicial o juvenil del rodal, denominada temprana, que se estableció hasta los 8 años, donde hay una marcada aceleración del incremento de la $\mathrm{Hd}$ en el cual se observa un crecimiento de 0,8 a $1 \mathrm{~m} / \mathrm{año}$; luego una etapa de edades intermedias, denominada media, entre los 8 a los 15 años, donde 
el incremento disminuye respecto a las edades anteriores, observando valores de incremento desde 0,4 a 0,8 m/año; y una etapa posterior, denominada avanzada, en la cual el rodal alcanza la altura final de aproximadamente 10 a $12 \mathrm{~m}$, en esta etapa se incluyen los rodales mayores a 15 años (Tabla 1).

La variabilidad de la Hd fue mayor en los rodales en la primera y segunda etapa de crecimiento (rango etario denominado temprano y medio) y, a medida que aumenta la edad del mismo, dicha variabilidad disminuye ( $\mathrm{DE}=1,6 \leq 15$ años y $1,1>15$ años) (Tabla 1). En concordancia con lo expresado por Herrera y Alvarado (1998), la mayor variabilidad que se presenta a edades tempranas responde al comportamiento de las masas influenciadas por factores como la variabilidad genética, las técnicas de plantación y la densidad inicial, que pueden ser clasificados como factores genéticos y factores de manejo silvícola.

\section{Curvas de crecimiento en altura dominante e índice de sitio}

La muestra de árboles evaluados en este trabajo se encuentra entre los 9 a 21 años de edad y un rango de alturas entre 6 a 10 m (Figura 2), coincidiendo con las máximas alturas registradas para la especie en bosques nativos, en un rango de 10 a $12 \mathrm{~m}$ de altura en edades avanzadas de $90 \mathrm{a}$ 100 años (Juárez de Galindez et al., 2005; Cisneros y Moglia, 2017). El patrón de comportamiento de la altura dominante $(\mathrm{Hd})$ en función de la edad de los individuos no refleja un patrón polimórfico que indique que poseen diferente forma para cada calidad de sitio, demostrando una tendencia de carácter anamórfico (Figura 2). Estos perfiles anamórficos llevaron a la decisión de la construcción de curvas anamórficas a partir de la curva guía.

El criterio para definir la edad base habitualmente es arbitrario y elegido en función de la situación (Thrower, 1986). Sin embargo, el mismo autor expresa que en especies del este y oeste de Norte América usualmente se definió la edad de base con relación a la longevidad de la especie. Así se

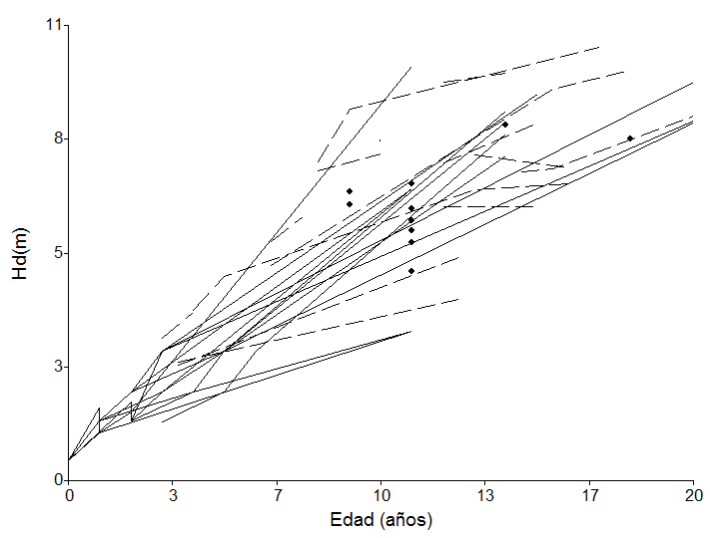

Figura 2. Patrón de comportamiento de la altura dominante en función de la edad en forestaciones de Prosopis alba, ubicadas en Santiago del Estero, Argentina. Línea continua: arboles evaluados a través de análisis de fuste; línea entrecortada: arboles evaluados en parcelas permanentes; puntos llenos: arboles relevados en parcelas temporales

estableció en 100 años para el caso de especies de larga vida, 50 años en especies de corta longevidad y 25 años o menos para plantaciones jóvenes o especies manejadas con rotaciones cortas (Thrower, 1986). Otro criterio es el expresado por Ortega y Montero (1988) quienes establecen que la edad base se encuentra próxima a la mitad del turno de corta final. En forestaciones de P. alba en la región del Chaco húmedo provincia de Chaco, Kees et al. (2017) proponen una edad base de 17 años y Pérez et al., (2011) para forestaciones con esta especie en la provincia de Formosa proponen como edad base 15 años. En este estudio la selección de la edad base en 15 años se considera adecuada, ya que según el registro de los datos, es la edad a la cual el potencial del sitio se ha expresado en el crecimiento en altura, coincidiendo aproximadamente con la mitad del turno de corta.

El modelo para obtener la curva guía y determinar el índice de sitio fue el modelo de regresión no lineal mixto de Gompertz con estructura de correlación simetría compuesta y función de varianza de los errores exponencial (Senilliani et al., 2019). El patrón de evolución en altura se ajusta satisfactoriamente al modelo Gompertz, adecuándose a los criterios biológicos

Tabla 1. Altura dominante $(\mathrm{Hd})$ promedio y variabilidad (DE, mínimo y máximo) con relación a la edad de los rodales, definida por rangos etarios en edades tempranas (de 1 a 8 años), edades medias (de 8 a 15 años) y edades avanzadas (más de 15 años) en rodales de Prosopis alba en Santiago del Estero

\begin{tabular}{lcccccc}
\hline Rango etario & Edad (años) & Parcelas (\#) & Hd media (m) & DE (m) & Hd mínima (m) & Hd máxima (m) \\
\hline Temprana & $\mathbf{1}$ a $\mathbf{8}$ & 47 & 2,6 & 1,6 & 1,0 & 7,7 \\
Media & $\mathbf{8}$ a 15 & 33 & 7,1 & 1,6 & 3,5 & 9,8 \\
Avanzada & $\mathbf{1 5}$ & 11 & 8,7 & 1,1 & 7,1 & 10,5 \\
\hline
\end{tabular}


de crecimiento. La aplicación de los modelos de efectos mixtos permitió modelar la estructura de los errores en datos provenientes de medidas repetidas y la presencia de heterocedasticidad. Los parámetros estimados en la función fueron estadísticamente significativos ( $p<0,0001)$.

La función es la siguiente:

$$
\mathrm{Hd}=9,1 \cdot \mathrm{e}^{\left(-2,3 \cdot \mathrm{e}^{(-0,2 \cdot \mathrm{E}))}\right.}
$$

donde, $\mathrm{Hd}=$ Altura dominante $(\mathrm{m})$ y $\mathrm{E}=$ edad (años). A partir de la ecuación [5] se graficó la curva guía que establece un IS de 8,2 metros a los 15 años (Figura 3a) y se pudo derivar la función que permitió expresar la familia de curvas anamórficas [6] que cubrían el espacio de crecimiento en altura dominante (Figura 3b).

a)

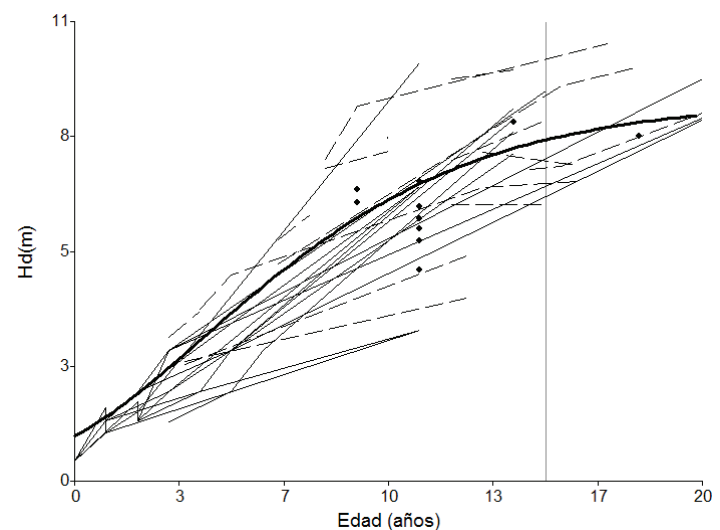

b)

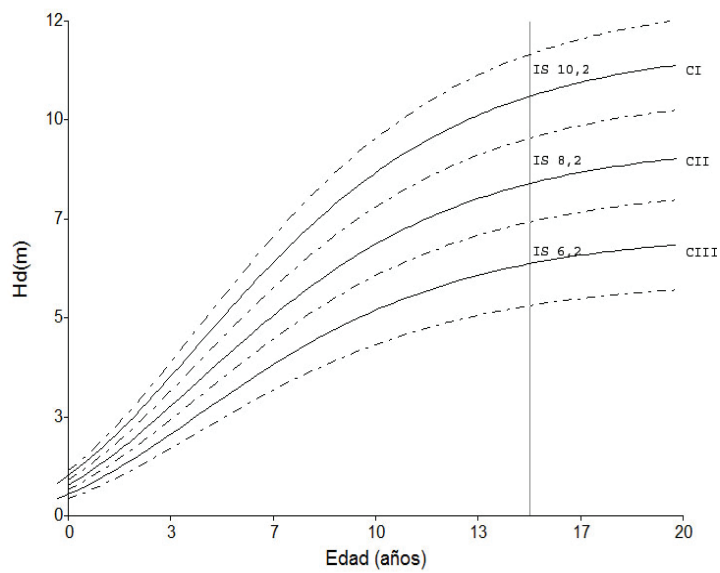

Figura 3. 3a) Perfiles de la Hd en función de la edad medida en años para estimar la curva guía a partir de la ecuación de Hd en función de la edad base de 15 años. La curva de crecimiento fue contrastada con series longitudinales de parcelas permanentes (líneas segmentadas), análisis de fustes (línea sostenida) y datos de parcelas temporales (líneas de puntos). 3b) Familia de curvas anamórficas derivadas de la primera (línea continua), describiendo el índice de sitio y los límites de cada clase (línea entrecortada)
La ecuación armonizada es:

$$
H d=I S . e^{\left(-\beta . e^{(-\gamma . E)}\right) / e^{(-\beta .)} \cdot(-\gamma .15)}[6]
$$

A partir de los datos del estudio se generó la familia de curvas anamórficas mediante la ecuación [6], coincidiendo con el procedimiento empleado por Barrero et al. (2011) y Hernández-Ramos et al. (2014). A partir de la curva guía obtenidas en este trabajo se establecieron tres clases de calidad de sitio que fueron establecidas en función al rango de alturas encontradas a la edad base, con una dispersión de los datos de Hd entre 6,5 a $10,5 \mathrm{~m}$. Los rangos de IS definidos cubren el espacio de crecimiento en altura dominante que tiene la especie. Los mismos se definieron de la siguiente forma: Clase I, correspondiente a la mejor calidad de sitio con $11,2 \geq I S \geq 9,2$; Clase II, correspondiente a una calidad de sitio intermedia con 9,2>IS $\geq 7,2$; y Clase III, que se corresponde con la calidad de sitio más baja con 7,2 $>$ IS $\geq 5,2$ (Figura 3b). A partir de la dispersión de los datos de altura dominante descripta anteriormente se estableció la equidistancia entre las curvas de IS de $2 \mathrm{~m}$ entre clases, determinándose de esta manera los valores de altura en función de la edad para cada una de las curvas de IS (Tabla 2). En el manejo forestal de la especie $P$. alba, conocer las clases de calidad en la zona de riego de la provincia es valioso para predecir o modelar la producción, como así también para hacer una correcta selección de los mejores sitios para plantación una vez determinados los factores del sitio que más influyan en el crecimiento.

Tabla 2. Valores predichos de altura dominante e índice de sitio según la clase de calidad de sitio (Cl, Cll y CIII) en función de la edad en plantaciones de Prosopis alba en la Región Chaqueña de la República Argentina

\begin{tabular}{cccc}
\hline & \multicolumn{3}{c}{$\mathbf{H d}(\mathbf{m})$} \\
\cline { 2 - 4 } Edad (años) & \multicolumn{3}{c}{ Índice de sitio } \\
\hline & $\mathbf{C I I I}^{*}$ & $\mathbf{C l I}^{\boldsymbol{*}}$ & $\mathbf{C l}^{\boldsymbol{*}}$ \\
& $\mathbf{6 , 2}$ & $\mathbf{8 , 2}$ & $\mathbf{1 0 , 2}$ \\
5 & 0,8 & 1,1 & 1,3 \\
$\mathbf{1 0}$ & 3,0 & 3,9 & 4,9 \\
15 & 5,2 & 6,9 & 8,6 \\
20 & 6,2 & 8,2 & 10,2 \\
25 & 6,5 & 8,6 & 10,7 \\
30 & 6,6 & 8,8 & 10,9 \\
& 6,7 & 8,8 & 11,0
\end{tabular}

${ }^{*} \mathrm{Cl}$ : calidad de sitio con IS $>9,2$; Cll: calidad de sitio con $7,2 \leq \mathrm{IS} \leq 9,2$ y CIII: calidad de sitio con IS $<7,2$

\section{Relación crecimiento-suelo}

La capacidad productiva de la especie está 
influenciada por el sitio de crecimiento; así lo expresa la Hd. El resultado del análisis de la varianza multivariado en relación con las propiedades físicas en el horizonte A, PTot (porosidad de la capa arable) y DA (densidad aparente) no registraron variaciones estadísticamente significativas a través de las clases de sitio. Si bien la porosidad (PTot) es una variable de importancia entre las propiedades físicas, registra para el horizonte analizado un rango de 49,9 a $53,6 \%$ sin diferencias estadísticamente significativas entre clases de calidad de sitio $(F=0,9 p=0,4)$. Los valores de PTot obtenidos en este trabajo fueron menores a los reportados por Meza Pérez et al. (2003) para bosques templados con comunidades de Pinus y Quercus en México (60 a 62\%), similares a los reportados por González-Barrios et al. (2011) y Mellado (2012) para bosques húmedos de coníferas en España (50\%) y superiores a los encontrados por Acuña (2012) en suelos de plantaciones con coníferas en áreas subtropicales de Perú (39 a 40\%). Se considera relevante poder determinar en futuros estudios la proporción según el tipo de poros, ya sea macroporos, mesoporos o microporos con funciones importantes en el balance de agua y aire y en el desarrollo de organismos (Lorenz, 2005).

El análisis de la varianza multivariado con las propiedades químicas $\mathrm{pH}, \mathrm{CE}$, RAS y cationes solubles $\left(\mathrm{Na}^{+}\right.$y $\left.\mathrm{Ca}+\mathrm{Mg}\right)$ presentó diferencias estadísticamente significativas entre clases de calidad de sitio en base al estadístico de Wilks $(0,5)(F=2,8 ; p=0,004)$. En la Tabla 3 se presentan los valores promedios para la $\mathrm{Hd}$ de los rodales y los respectivos valores de las variables edáficas evaluadas según la clase de sitio.

Las propiedades químicas a diferencia de las físicas, son determinantes, presentando diferencias estadísticamente significativas entre clases como se expresó anteriormente. Sin embargo, en comparaciones a posteriori entre clases, se observa que las clases I y II no difieren estadísticamente entre sí (Clase I vs Clase II, valor $p=0,67$ ) mientras que sí difieren ambas con respecto a la clase III (Clase I vs Clase III, valor $\mathrm{p}<0,001$ y Clase II vs Clase III, valor $\mathrm{p}=0,001$ ). Estos resultados sugieren que si bien los cambios registrados en las propiedades químicas entre las clases I y II no son estadísticamente significativos, la $\mathrm{Hd}$ de los rodales estudiados difiere entre estas clases, siendo sus diferencias estadísticamente significativas. Esta diferencia registrada en la $\mathrm{Hd}$ pero no en las propiedades químicas podría deberse a la sensibilidad de la especie al crecimiento ante pequeños incrementos en la salinidad, resultando en una disminución de su crecimiento en altura. También sería posible considerar que los cambios en Hd que experimenta la especie de la clase I a la II se deban a otros factores no considerados en este estudio.

Considerando la clase I y II se propone como valores indicadores para la CE valores de 5,4 a $7 \mathrm{dSm}^{-1}$ al igual que la concentración de sodio de hasta aproximadamente 39 meg/l. Esta estimación se realiza considerando que estas clases, donde se registra el valor medio de IS de 10,2 y 8,2 , son consideradas las clases de mayor producción maderera. El RAS no supera el valor de 8,8 para la clase I y II; sin embargo, en la clase III los valores llegan a 22. Esto indica una creciente relación de adsorción de sodio en la solución del suelo de la clase I a la III con una consecuente disminución de la capacidad potencial de crecimiento de los

Tabla 3. Valores promedios y desviación estándar (DE) para la variable dasométrica Hd y propiedades químicas y físicas del suelo por clase de calidad de sitio (la muestra por clase es igual a $n=12$ en la clase I, $n=25$ en clase II y $n=8$ en clase III). Los valores corresponden al horizonte $\mathrm{A}$ en el perfil del suelo

\begin{tabular}{|c|c|c|c|}
\hline & \multicolumn{3}{|c|}{ Clase de calidad de sitio } \\
\hline & Clase I & Clase II & Clase III \\
\hline \multicolumn{4}{|l|}{ Variable dasométrica } \\
\hline $\mathrm{Hd}(\mathrm{m})$ & $9,3 \pm 0,07 a$ & $8,0 \pm 0,78 b$ & $6,8 \pm 0,35 c$ \\
\hline \multicolumn{4}{|l|}{ Variables químicas } \\
\hline $\mathrm{pH}$ & $7,9 \pm 1,1 \mathrm{a}$ & $8,1 \pm 0,7 a$ & $8,1 \pm 1,0 \mathrm{a}$ \\
\hline $\mathrm{CE}\left(\mathrm{dSm}^{-1}\right)$ & $5,4 \pm 2,7 a$ & $7,0 \pm 5,0 a$ & $19,9 \pm 16,0 b$ \\
\hline $\mathrm{Ca}+\mathrm{Mg}(\mathrm{meq} / \mathrm{l})$ & $38,7 \pm 32,9 a$ & $44,5 \pm 32,8 a$ & $83,9 \pm 61,5 b$ \\
\hline $\mathrm{Na}^{+}(\mathrm{meq} / \mathrm{l})$ & $21,5 \pm 12,1 \mathrm{a}$ & $39,6 \pm 39,8 a$ & $137,4 \pm 108,2 b$ \\
\hline RAS (meq/l) & $6,2 \pm 4,5 a$ & $8,8 \pm 7,5 a$ & $22,1 \pm 11,0 b$ \\
\hline \multicolumn{4}{|l|}{ Variables físicas } \\
\hline $\mathrm{DA}\left(\mathrm{g} / \mathrm{cm}^{3}\right)$ & $1,1 \pm 0,1 a$ & $1,1 \pm 0,1 a$ & $1,2 \pm 0,1 a$ \\
\hline PTot (\%) & $52,4 \pm 3,5 a$ & $53,6 \pm 5,5 a$ & $49,9 \pm 6,5 a$ \\
\hline
\end{tabular}

Letras iguales para cada fila simbolizan que las medias no son significativamente diferentes $(p>0,05)$ 
rodales. En la variable concentración de $\mathrm{Ca}+\mathrm{Mg}$, el comportamiento es similar; sin embargo, el pH no muestra diferencias entre clases (Tabla 3 ). En la variable presencia de carbonatos $(p<0,1)$ no se observaron valores que difieran entre las clases. Ante las características geomorfológicas de los suelos de la región es notable la relevancia que tienen las variables químicas del suelo en contraposición a otros sitios de estudio como la provincia del Chaco donde el $58 \%$ de las causas de variación de $\mathrm{Hd}$ en plantaciones de $P$. alba se relaciona a variables fisiográficas (relieve, drenaje) y edáficas (textura) mientras que las variables químicas del suelo no explicaron diferencias en la calidad de sitio (Kees et al., 2017).

A partir de los resultados encontrados en este trabajo, se puede inferir que el crecimiento está condicionado negativamente por parámetros característicos de las condiciones de salinidad en los suelos. Las propiedades químicas evaluadas en los suelos de las plantaciones de $P$. alba en estudio demuestran ser un factor de importancia e impacto en la productividad de la especie.

\section{Validación del modelo}

El modelo ajustado para la altura dominante $(\mathrm{Hd})$ indicó que en cada una de las clases de calidad de sitio y a edades tempranas se pueden presentar algunos valores extremos de diferencia de altura que pueden llegar a más de $1 \mathrm{~m}$, pero en general a partir de los 10 años el error es menor, decreciendo el sesgo de la estimación a edades intermedias para las tres clases. La variabilidad en la estimación a edades medias para las clases presenta un rango de error de 0,2 a 1,1 y la tendencia es a sobreestimar en la clase I y a subestimar en la clase II. La clase III a partir de los 11 a 12 años muestra una tendencia a sobreestimar el IS dentro del rango de error nombrado. En general se puede concluir que el error estaría en un rango del 5 al $10 \%$ para la edad base de 15 años, estimada en este trabajo. En concordancia con estudios realizados sobre otras especies, diferentes autores (Amico et al., 2010; Ribeiro et al., 2016) evaluaron la precisión de los modelos de índice de sitio como satisfactoria con valores similares que no superan el $10 \%$ RECM. Se visualizaron diferencias en la estimación de las alturas, enunciadas anteriormente, de hasta $1 \mathrm{~m}$ y un alejamiento máximo de un valor observado en relación a la curva de clase I de $2 \mathrm{~m}$ a la edad de 9 años (Figura 4).

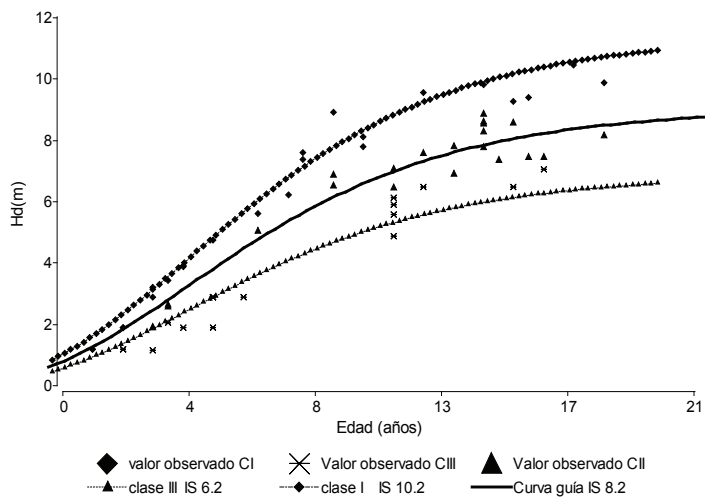

Figura 4. Variabilidad de la estimación entre los valores estimados (curva guía y curvas correspondientes a clase II y III) y los valores observados (rombos, triángulos y asteriscos) de las alturas dominantes promedio en relación a la edad, correspondientes a cada clase de calidad de sitio (C I, C II y CIII) respectivamente

\section{CONCLUSIONES}

El patrón de evolución en altura muestra que la especie culmina su crecimiento a edades tempranas en plantaciones del área de estudio, cercanas a los 20 años, si bien esto deberá corroborarse con estudios a edades más avanzadas. Una primera aproximación en la estimación de la capacidad productiva de los sitios para P. alba Griseb se expresa a partir de la familia de curvas anamórficas a la edad base de 15 años, representativas de tres calidades de sitio, I (buena), II (regular) y III (mala). El proceso de validación determinó que el error estaría en un rango del 5 y $10 \%$ para la edad base (15 años). Los factores del sitio que son determinantes en el comportamiento de las clases de calidad son los factores edáficos a través de las propiedades químicas del suelo que a su vez describen las condiciones de salinidad.

La caracterización de los sitios de plantación de $P$. alba y la estimación del índice de sitio en el área de riego de la provincia es una herramienta importante para poder realizar una correcta selección de un sitio para plantación. Ante lo cual se recomienda para algarrobo blanco, tener en consideración los valores indicadores de CE y concentración de cationes solubles de sodio, los cuales no deberían superar los 5 a $7 \mathrm{dS} \mathrm{m}^{-1} \mathrm{y}$ $39 \mathrm{meq} / \mathrm{l}$ respectivamente. Se considera necesario profundizar el estudio con la ampliación de la base de datos, aumentar la muestra de estudio para la evaluación potencial de los sitios incorporando mayor número de mediciones en parcelas permanentes de diferentes edades y sitios e incorporar, si se justificara, el análisis de patrones polimórficos, que podría conferir mayor precisión 
en la estimación de las calidades de sitio.

Para avanzar en una adecuada zonificación de la actividad de plantación con $P$. alba se recomiendan las calidades de sitio I y II para forestación con fines de obtención de servicios de provisión (madera y frutos) y la calidad III, debido a su baja productividad, podría destinarse a la producción de frutos y servicios ecosistémicos de regulación, entre ellos la recuperación de áreas degradadas.

\section{BIBLIOGRAFÍA}

Alder, D. (1980). Forest volumen estimation and yield prediction (Vol.2), FAO Forestry.

Assman, E. (1970). The Principles of Forest Yield Study. Pergamon Press. https://doi.org/10.1016/B978-0-08006658-5.50002-9

Acuña, M. (2012) Efecto de las plantaciones de Pinus sobre las propiedades del suelo Cullpa alta, Huancayo, Junin. Tesis de grado no publicada. Universidad nacional del Centro de Perú. Huancayo, Perú.

Amico, I., Bava, J. y Calderon, A. (2010) Índices de calidad de sitio de Populus nigra'ltalica' en plantaciones lineales en el noroeste de Chubut. Revista de la Facultad de Ciencias Agrarias. Universidad Nacional de Cuyo. 42(1), 147-158. http://www.redalyc.org/ articulo.oa?id=382837646010

Andenmatten, E. y Letourneau, F. (1998). Curvas de índice de sitio y crecimiento en altura para pino oregón (Pseudotsuga menziesii) (Mirb.) Franco de aplicación en la Región Andino Patagónica de las provincias de Río Negro y Chubut, Argentina. Revista de la Facultad de Agronomía .Universidad Nacional de La Plata, 103(1), 61-68. http://revista.agro.unlp.edu.ar/index. php/revagro/article/view/239

Barrero-Medel, H., Mothe, F., Nepveu, G., Álvarez Lazo, C., García-Corona I. y Guera, M. (2011). Curvas anamórficas de índice de sitio para plantaciones de Pinus caribaea Morelet var. Caribaea Barret y Golfari de la empresa forestal integral macurije (EFI) en la provincia de Pinar del Río, Cuba. Chapingo, Serie Ciencias Forestales y del Ambiente. 17(2), 245-252. https://doi.org/10.5154/r.rchscfa.2010.06.035

Barret, J. (1978). Height growth and site index curves for managed, even-aged stands of Ponderosa pine in the Pacific Northwest. U.S. Department of Agriculture, Forest Service, Pacific Northwest Research Station. https://www.fs.usda.gov/treesearch/pubs/37176

Caniza, F. J. y Torres, C. G., (17,18 y 19 de octubre 2019). Funciones de Índice de Sitio para Pinus taeda en las Planicies Arenosas de Corrientes, Argentina. Documento presentado en Jornadas Forestales 2019.
Eldorado, Misiones, Argentina.

Caniza, F. J., Torres, C. G. y Aparicio, J. L. (2016). Desarrollo de funciones de índice de sitio para Eucalyptus grandis en las Planicies Arenosas de Corrientes, Argentina. En Facultad de Ciencias Forestales UNAM (Eds.), XVII Jornadas Técnicas forestales y ambientales (pp 131-133). Posadas, Misiones. Argentina.

Cisneros, A. y Moglia, J. G. (2017). Prosopis alba, alternativa sustentable para zonas áridas y semiáridas. En A. M. Giménez y J. G. Moglia. (Eds.), Los bosques actuales del Chaco semiárido argentino. Ecoanatomía y biodiversidad. Una mirada propositiva (231-248). Universidad Nacional de Santiago del Estero. Facultad de Ciencias Forestales.

Clutter, J., Fortson, J., Pienaar, L., Brister, G. y Bailey, R. (1983). Timber Management: A Quantitative Approach. Wiley.

Crechi, E., Fassola, H., Keller, A. y Barth, S. (2011) Desarrollo de funciones de índice de sitio para Eucalyptus grandis cultivado en la Mesopotamia Argentina. Revista de Investigaciones Agropecuarias, 37 (3), 238-248. http://www.redalyc.org/articulo. oa?id=86421245008

Di Rienzo, J., Casanoves, F., Balzarini, M., González, L., Tablada, M. y Robledo, C. InfoStat (versión 2020). [Software] Córdoba, Argentina: Grupo InfoStat, FCA, Universidad Nacional de Córdoba, Argentina. http:/l www.infostat.com.ar.

Dirección Nacional de Desarrollo Foresto Industrial, Ministerio de Agroindustria de la Nación Argentina (2015). Informe Nacional del relevamiento censal de aserraderos 2015. https://www.google.com/ search?client=firefox-b-d\&q=INFORME+NACIONAL+ DELRELEVAMIENTO+CENSAL+DEASERRADEROSA $\%$ C3\%91O+2015

Efron, B. y Tibshirani, R. (1993) An Introduction to the Bootstrap. Chapman and Hall.

Fassola, H. y Wabo, E. (1993). Funciones de calidad de sitio para Pinus elliottii Engelm en Misiones (Argentina). Revista Yvyraretá 4 (4), 26 - 34.

GeolNTA (2017) SAGyP- INTA - Proyecto PNUD ARG/85/019. (Versión 9.0)

Giménez, A., Ríos, N., Moglia, J. y López, C. (1998). Leño y corteza de Prosopis alba Griseb., algarrobo blanco, Mimosaceae, en relación con algunas magnitudes dendrométricas. Bosque 19(2), 53-62. https://doi. org/10.4206/bosque.1998.v19n2-06_

Giménez, A., Ríos, N., Moglia, J., Hernández, P., y Bravo, S. (2001). Estudio de magnitudes Dendrométricas en función de la edad en Prosopis alba Griseb., Mimosaceae. Revista Forestal Venezolana 45(2), 175183. 
González-Barrios J., González-Cervantes. G., SánchezCohen I., López-Santos A. y Valenzuela-Núñez L. (2011). Caracterización de la porosidad edáfica como indicador de la calidad física del suelo. Terra Latinoamericana, 29(4), 369-377. http://www.redalyc. org/articulo.oa?id=57322342002

Hernández-Ramos, J., García-Magaña, J., García Cuevas, X., Hernández-Ramos, A., Muñoz Flores, H. y Samperio-Jiménez, M. (2014). Índice de sitio para bosques naturales de Pinus teocote Schlecht. \&Cham en el oriente del estado de Hidalgo. Revista Mexicana de Ciencias Forestales. 6(27), 24-37. http://www.scielo. org. $\mathrm{mx} /$ scielo. php?script=sci_arttext\&pid=S2007$11322015000100003 \&$ lng =es\&tlng=es

Herrera, B. (1996). Evaluación del efecto del sitio en la productividad de las poblaciones de dos especies dominantes en un bosque tropical de la tercera fase de la sucesión secundaria en Costa Rica. Tesis Magister Scientiae no publicada, Centro Agronómico Tropical de Investigación y Enseñanza, Turrialba, Costa Rica. https://repositorio.catie.ac.cr/discover

Herrera, B. y Alvarado, A. (1998). Calidad de sitio y factores ambientales en Bosques de Centro América. Agronomía Costarricense 22(1), 99-117.

Juárez de Galindez, M., Giménez, A., Ríos, N. y Balzarini, M. (2005). Modelación de crecimiento en Prosopis alba Griseb empleando dos modelos biológicos. Revista Quebracho, 12, 34-42.

Kees, S., Ferrere, P., Lupi, A., Michela, J. y Skoko, J. (2018). Producción y crecimiento de las plantaciones de Prosopis alba Griseb en la provincia del Chaco. Revista de Investigaciones Agropecuarias, 44(2), 113-120. https://www.redalyc.org/journal/864/86457304010/ $\mathrm{html} /$

Klute A. (Ed.) (1986) Methods of soils analysis. Part 1 Physical and mineralogical methods. American Society of Agronomy.

Ledesma, T., de Bedia, G. y López, C. (2008). Productividad de Prosopis alba Griseb en Santiago del Estero. Quebracho, 15, 5-8

Lorenz, G. (2005) Métodos de análisis de suelos. (Serie didáctica No12) Cátedra de Edafología Forestal. Manuscrito no publicado, Universidad Nacional de Santiago del Estero, Facultad de Ciencias, Forestales.

Mares Arreola, O., Cornejo Oviedo, E., Valencia Manzo, S. y Flores López, C. (2004). Índice de sitio para Pinus Herrarae Martinez en Cd. Hidalgo, Michoacán. Revista Fitotecnia Mexicana, 27(Num. Especial 1), 7780 https://www.redalyc.org/articulo.oa?id=61009916

Mellado, T., (2012). Influencia de los parámetros edáficos, topográficos y climáticos sobre el índice de sitio de Pinus sylvestris L. en las masas de León y Palencia. Tesis de Maestría Universidad de Valladolid, España. http://uvadoc.uva.es/handle/10324/1548
Meza Pérez, E. y Geissert Kientz, D. (2003). Estructura, agregación y porosidad en suelos forestales y cultivados de origen volcánico del Cofre De Perote, Veracruz, México. Foresta Veracruzana, 5(2), 57-60. https://www.redalyc.org/articulo.oa?id=49750209

Morello, J., Matteuci, S., Rodriguez, A, Silva, M. (2012). Ecorregiones y complejos ecosistémicos argentinos. Orientación Grafica Editora.

Navall, M., Ewens, M., Senilliani, M. y López, C. (2015). Efectos de la poda en plantaciones jóvenes de Prosopis alba Griseb en Santiago del Estero, Argentina. Quebracho, 23 (2), 77-91.

Organización de las Naciones Unidas para la Alimentación y la Agricultura (FAO) (2009). Guía para la descripción de suelos ( $4^{a}$ ed.). FAO

Ortega, A. y Montero, G. (1988). Evaluación de la calidad de sitio de las estaciones forestales. Revisión bibliográfica. Ecología, 2, 155-184. https://www. miteco.gob.es/ministerio/pags/Biblioteca/Revistas/ pdf_REPN\%2FREPN_1988_155-184.pdf

Pérez, V., Vicentini, G., Ocampo, L. y Vega, C. (4-9 de abril 2011). Evaluación preliminar de la calidad de sitio para Prosopis alba Griseb mediante la utilización de criterios dasométricos. En $1^{\circ}$ Congreso Forestal del Chaco Sudamericano. Producción y Sustentabilidad (pp.21-30). Boquerón, Paraguay.

Prodan, M., Peters, R., Cox, F. y Real Hermosilla, P. (1997.) Mensura Forestal. Instituto Interamericano de Ciencias Agrícolas.

R Core Team (2020). R: A language and environment for statistical computing. R Foundation for Statistical Computing, Vienna, Austria. URL https://www.Rproject.org

Red Agroforestal Chaco Argentina (RedAF) (1999). Estudio Integral de la Región del Parque Chaqueño. (Informe General Ambiental Red Agroforestal Chaco Argentina. Proyecto Bosques Nativos y Áreas Protegidas Préstamo BIRF No 4085). RedAF.

Ribeiro, A., Ferraz Filho, A., Tomé, M. y Soares Scolforo, J. (2016). Site quality curves for African mahogany plantations in Brazil. CERNE, 22(4), 439-448. https:// doi.org/ 10.1590/01047760201622042185

Salto, C., Harrand, L., Oberschelp, G. y Ewens, M. (2016).Crecimiento de plantines de Prosopis alba en diferentes sustratos, contenedores y condiciones de vivero. Bosque 37(3), 527-537. https://doi.org/10.4067/ S0717-92002016000300010

Sarmiento, G. (1963). Las comunidades vegetales del Chaco semiárido santiagueño. Tesis Doctoral no publicada, Facultad de Ciencias Exactas y Naturales, Universidad de Buenos Aires.. http://hdl.handle. net/20.500.12110/tesis_n1243_Sarmiento

Schlatter, J. y Gerding, V. (2014). Sitio Forestal. En C. 
Donoso, M.E González, A. Lara (Eds.), Ecología forestal. Bases para el manejo sustentable y la conservación de los bosques nativos de Chile. (pp.309-319) Valdivia, Chile Ediciones UACh p. 309319.

Senilliani, M. G., Bruno, C. y Brassiolo, M. (2019). Site index for Prosopis alba plantations in the semi-arid Chaco through mixed models. CERNE 25(2), 195-202. https://doi.org/ 10.1590/01047760201925022622

Sparks, D. L. (Ed.). (1996). Methods of Soil Analysis. Part
3: Chemical methods. Soil Science Society of America, American Society of Agronomy.

Soil Survey Staff (2014). Keys to Soil Taxonomy (12a ed.). USDA-Natural Resources Conservation Service.

Thrower, J. (1986). Estimating site quality from early height growth of white spruce and red pine plantations in the Thunder Bay area. Tesis de maestría no publicada. Lakehead University, Ontario. http:// knowledgecommons.lakeheadu.ca/handle/2453/2161 